\title{
Optimization of virtual power plant with a distributed generation
}

\author{
Elena Sosnina ${ }^{1}$, Andrey Shalukho ${ }^{1, *}$, and Alexander Kechkin ${ }^{1}$ \\ ${ }^{1}$ NNSTU n.a. R.E. Alekseev, 603950 N. Novgorod, Russia
}

\begin{abstract}
The article is devoted to the task of optimizing the structure of virtual power plants (VPP) with distributed generation sources. The solution of the problem is based on the structural topological and probabilistic-statistical analysis of the VPP structure. An algorithm for estimating the structure of VPP has been developed. The optimization criteria for VPP are proposed and investigated. The criteria allow to evaluate VPP structural properties and to take into account the inconstancy of generation from renewable energy sources (RES) in VPP composition.
\end{abstract}

\section{Introduction}

An important direction in the development of distributed energy is connected with a merger of various small sources of generation (SSG) with energy storage device and consumers in the VPP [1-3]. In this case the problems of choosing the optimal structure of VPP are modern and research require. For evaluation the efficiency and reliability of systems with SSG widely indications apply: voltage level, loss of power, short circuit currents, SAIDI and SAIFI characteristics. However to constitutive properties of grid and existence of equivocations not enough attention have given and this can lead to the adoption of suboptimal solutions.

The authors investigate the solution of optimization VPP problems which based on structurally topological and probabilistic statistical analysis of VPP structure.

\section{Materials and methods}

Evaluation algorithm of VPP structure was developed by using the theory of graphs. The algorithm consists of three stages.

Stage 1 - electrotechnical complex of VPP is represented as an oriented graph [4]. It is possible to build several graphs for analysis which reflect the various options for electrical connections in VPP.

Stage 2 - the considered graphs are represented in matrix form, are calculated structurally topological graph characteristics: structural connectivity, structural redundancy, uneven distribution of connections, structural compactness, degree of centralization [5].

Structural connectivity $(c)$ :

* Corresponding author: shaluho.andrey@mail.ru 


$$
c=\sum_{i=1}^{n} \sum_{j=1}^{n} c_{\mathrm{ij}}
$$

where $c_{\mathrm{ij}}=1$, if $a_{\mathrm{ij}} \geq 1$ and $c_{\mathrm{ij}}=0$, if $a_{\mathrm{ij}}=0\left(a_{\mathrm{ij}}\right.$ is the total number of paths from vertex $i$ to vertex $j$ ).

The larger the value of $c$, the structure is more connected and potentially more reliable.

Structural redundancy $(R)$ :

$$
R=\left[\sum_{i=1}^{n} \sum_{j=1}^{n} a_{\mathrm{ij}}\right] \cdot \frac{1}{n-1}-1,
$$

where $a_{\mathrm{ij}}$ is the element of the adjacency matrix of vertices $A, n$ is the number of vertices of the graph.

The minimum value of $R$ indicates the smallest number of excess electrical connections that increase the cost of power lines and equipment.

Uneven distribution of connections $\left(\varepsilon^{2}\right)$ :

$$
\varepsilon^{2}=\sum_{i=1}^{n}\left(\rho_{\mathrm{i}}^{2}-\frac{4 m^{2}}{n}\right)
$$

where $\rho_{\mathrm{i}}$ is the number of edges incident to the $i$-th vertex of the graph, $m$ is the number of edges of the graph.

The structure with the minimum value $\varepsilon^{2}$ is considered to be the most effective.

Structural compactness $(Q)$ :

$$
Q=\sum_{i=1}^{n} \sum_{j=1}^{n} d_{\mathrm{ij}}
$$

where $d_{\mathrm{ij}}$ - is the minimum path length from vertex $i$ to vertex $j$.

Structures with a smaller value of $Q$ are characterized by potentially smaller losses and a greater degree of efficiency in management.

Degree of centralization $(\delta)$ :

$$
\delta=(n-1) \frac{2 Z_{\mathrm{MAX}}-n}{Z_{\mathrm{MAX}}(n-2)},
$$

where $Z_{\mathrm{MAX}}$ is the value determined from the calculation of structural compactness.

A high value of $\delta$ places high demands on the capacity of the central element.

Stage 3 - using the weighted sums of criteria method, an integral evaluation of the VPP structures being compared is performed:

$$
K_{\mathrm{j}}=\sum_{i=1}^{M} k_{\mathrm{i}} P_{\mathrm{ij}}, i=1, \ldots M, j=1, \ldots N,
$$

where $N$ is the number of compared structures, $M$ is the number of compared characteristics $\left(c, R, \varepsilon^{2}, Q, \delta\right), P_{\mathrm{ij}}$ is the estimated estimate of the $i$-th characteristic of the $j$-th structure, $k_{\mathrm{i}}$ is the coefficient of value of the $i$-th characteristic. 
The most effective structure is considered to be the VPP, to which the largest value of $K_{\mathrm{j}}$ corresponds.

\section{Result and discussion}

Consider an example of the integration of two IMGs (wind power plant (WPP) and gas piston unit (GPU)) into the power supply system of consumers receiving power from a centralized electrical network.

A simplified single-line diagram and the obtained graph of the distributed electric network are shown in Fig. 1 and Fig. 2. For 13 possible structures structural-topological characteristics (1) - (5) were calculated and their integral estimation was performed.

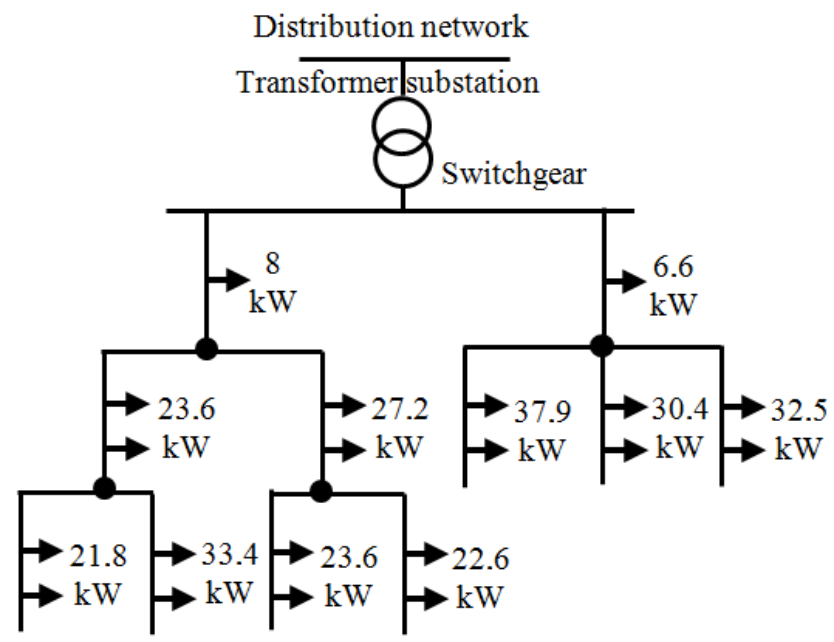

Fig. 1. Simplified single-line diagram of a distributed electrical network.

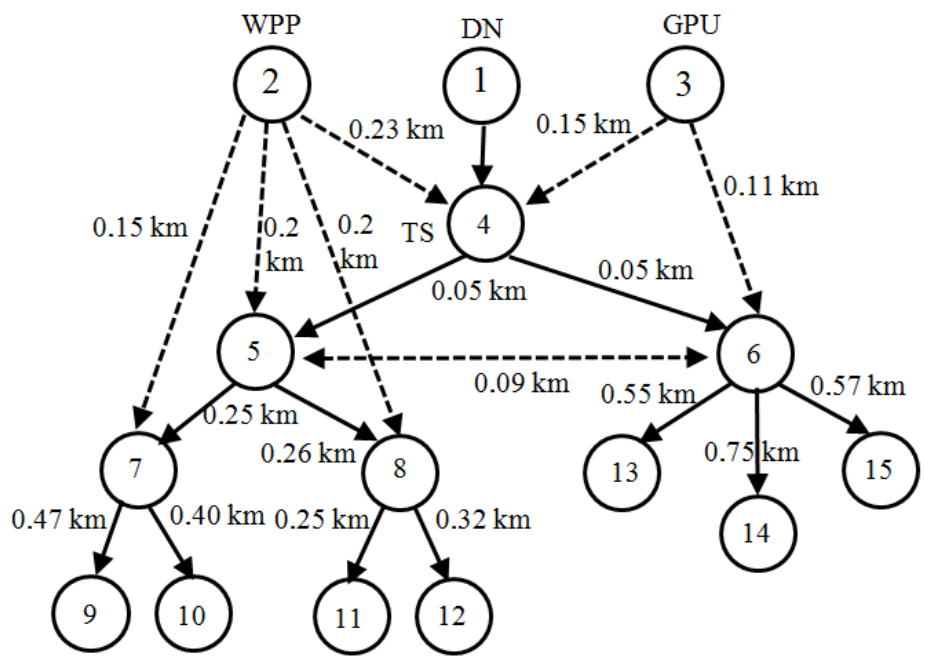

Fig. 2. Graph of a distributed electrical network.

The results of calculations for two variants of the structure with the largest values of $K_{\mathrm{j}}$ are given in Table 1. 
Table 1. Results of structural topological analysis.

\begin{tabular}{|c|c|c|c|c|c|c|}
\hline Option structure & $c$ & $R$ & $\varepsilon^{2}$ & $Q$ & $\delta$ & $K_{\mathrm{j}}$ \\
\hline $\mathrm{K} 1$ & 50 & 0 & 37.8 & 103 & 1.429 & 0.958 \\
\hline $\mathrm{K} 2$ & 50 & 0 & 37.8 & 103 & 1.429 & 0.958 \\
\hline
\end{tabular}

The WPP is connected to the node 7 in the variant $\mathrm{K} 1$, and in the variant $\mathrm{K} 2$ to the node 8. The GPU is connected to the node 6 in both versions of the structure.

For a more detailed analysis of the selected version of the structure of the VPP, in addition to calculating technical and economic indicators, it is proposed to use the ranking of its elements. The most significant elements in the structure of the VPP are determined. To assess the significance, the "element rank" parameter can be used [6]:

$$
r_{\mathrm{i}}=\sum_{j=1}^{n} a_{\mathrm{ij}}^{\mathrm{k}} / \sum_{i=1}^{n} \sum_{j=1}^{n} a_{\mathrm{ij}}^{\mathrm{k}}
$$

where $a_{\mathrm{ij}}$ is an element of the adjacency matrix, $k$ is the path length from vertex $i$ to vertex $j$.

The higher the rank of the element, the more severe the consequences will be if its functioning is disrupted. The results of calculating the ranks of the elements for the selected VPP structures are shown in Table 2.

Table 2. Results of calculating the ranks of the elements.

\begin{tabular}{|c|c|c|c|c|c|}
\hline \multirow{2}{*}{ Structure } & \multicolumn{5}{|c|}{ Elements of the graph and their ranks } \\
\cline { 2 - 6 } & 1 & 2 & 3 & 4 & $5-16$ \\
\hline K1 & 0.64 & 0 & 0 & 0.36 & 0 \\
\hline K2 & 0.64 & 0 & 0 & 0.36 & 0 \\
\hline
\end{tabular}

For both structures, the key elements are nodes 1 and 4. For more complex systems, the uniform distribution of the participation of elements in the formation of the structure is considered to be the best. When renewable energy sources (RES) are part of the VPP structure, it is important to take into account the volatility of their generation. The inconstancy of RES generation is proposed to be assessed using a special criterion - $R_{\text {RES}}$.

Under $R_{\text {RES }}$ is the probability that within a certain time interval (for example, a month) the consumer will use the entire amount of RES produced. It is assumed that the distribution of generated electricity corresponds to the normal law. In this case, the $R_{\mathrm{RES}}$ value is determined by solving the integral function of the normal distribution [6]:

$$
R_{\mathrm{RES}}=\int_{-\infty}^{W_{\mathrm{CE}}} \frac{1}{\sqrt{2 \pi} \sigma\left(W_{\mathrm{RES}}\right)} \mathrm{e}^{-\frac{\left(W_{\mathrm{RESi}}-\mu\left(W_{\mathrm{RES}}\right)\right)^{2}}{2 \sigma\left(W_{\mathrm{RES}}\right)^{2}}} \mathrm{~d} W
$$

where $W_{\mathrm{CE}}$ is the amount of consumed electricity, $W_{\mathrm{RESi}}$ is the generated electricity of RES, $\mu\left(W_{\mathrm{RES}}\right)$ is the mathematical expectation of the renewable electricity produced, $\sigma\left(W_{\mathrm{RES}}\right)$ is the standard deviation of the generated electricity. The calculation of $R_{\mathrm{RES}}$ for connection of WPP to nodes 7 and 8 has been performed (Table 3 ). 
Table 3. The probability of consumption of the produced electric power by WPP.

\begin{tabular}{|c|c|c|c|c|c|c|c|c|c|c|c|c|c|}
\hline \multirow{2}{*}{ Node } & $\begin{array}{c}\text { Time } \\
\text { of } \\
\text { day }\end{array}$ & 1 & 2 & 3 & 4 & 5 & 6 & 7 & 8 & 9 & 10 & 11 & 12 \\
\cline { 3 - 15 } & Day & 69.5 & 74.9 & 65.5 & 35.6 & 50.8 & 46.8 & 72.6 & 64.4 & 79.1 & 81.3 & 97.5 & 53.6 \\
\cline { 2 - 14 } 7 & Night & 54.8 & 58.7 & 59.5 & 62.9 & 99.6 & 95.4 & 96.9 & 85.8 & 99.0 & 94.2 & 92.1 & 67.4 \\
\hline \multirow{2}{*}{8} & Day & 80 & 85.1 & 77.3 & 42.5 & 59.1 & 55.2 & 81.1 & 73.2 & 88.3 & 88.5 & 99.6 & 59.9 \\
\cline { 2 - 13 } & Night & 53.6 & 56.8 & 57.5 & 57.5 & 98.6 & 91.3 & 92.9 & 80.8 & 97.6 & 92.8 & 90.5 & 65.2 \\
\hline
\end{tabular}

From the results it follows that it is necessary to provide for the possibility of transferring electric power from the VPP to neighboring nodes with a load.

For VPP with a large number of aggregated objects, an important criterion for optimization can be an assessment of the chaotic nature of processes. The VPP can be characterized as a dynamic dissipative nonlinear system [7]. It is known that chaotic processes are typical for such systems. The danger of chaotic regimes is that they can lead to the system's exit from a steady state and an accident. Therefore, the chaotic processes in the VPP should be studied with a view to a deeper understanding of the mechanisms for ensuring the sustainability of the VPP.

\section{Conclusion}

In conclusion, it can be noted that the proposed and investigated optimization criteria for the VPP allow us to evaluate the structural properties of the VPP and take into account the volatility of the generation of renewable energy.

The work was carried out with the financial support of the Ministry of Education and Science of the Russian Federation (state task No. 13/2078/2017/ПЧ).

\section{References}

1. E.N. Sosnina, A.V. Shalukho, A.Yu. Kechkin, Vestnik NNSEEI, 4, 50 (2015)

2. E. Sosnina, I. Lipuzhin, A. Shalukho, A. Kechkin, IJAER, 10, 44363 (2015)

3. E.N. Sosnina, A.V. Shalukho, I.A. Lipuzhin, A.Yu. Kechkin, Vestnik SSTU Tech. Sci. Series, 2, 121 (2017)

4. E.N. Sosnina, A.V. Shalukho, A.Yu. Kechkin, N.V. Shumsky, Operative manage. electrical power-engine., 4, 48 (2017)

5. N.I. Voropay, The theory of systems for electric power engineering specialists (Novosibirsk, 2010) [in Russian]

6. E.N. Sosnina, A.V. Shalukho, Power Tech. Engine., 6, 467 (2013)

7. K.S. Demirchyan, Power Engine. Transp., 3, 3 (1987) 